University of Nebraska - Lincoln

DigitalCommons@University of Nebraska - Lincoln

\title{
Risk Factors Predictive of the Problem Behavior of Children At Risk for Emotional and Behavioral Disorders
}

\author{
J. Ron Nelson \\ University of Nebraska - Lincoln, rnelson8@unl.edu \\ Scott Stage \\ University of Washington, scott_stage@ncsu.edu \\ Kristin Duppong-Hurley \\ University of Nebraska-Lincoln, kristin.hurley@unl.edu \\ Lori Synhorst \\ University of Nebraska-Lincoln \\ Michael H. Epstein \\ University of Nebraska-Lincoln, mepstein1@unl.edu
}

Follow this and additional works at: https://digitalcommons.unl.edu/specedfacpub

Part of the Special Education and Teaching Commons

\begin{abstract}
Nelson, J. Ron; Stage, Scott; Duppong-Hurley, Kristin; Synhorst, Lori; and Epstein, Michael H., "Risk Factors Predictive of the Problem Behavior of Children At Risk for Emotional and Behavioral Disorders" (2007). Special Education and Communication Disorders Faculty Publications. 32.

https://digitalcommons.unl.edu/specedfacpub/32
\end{abstract}

This Article is brought to you for free and open access by the Department of Special Education and Communication Disorders at DigitalCommons@University of Nebraska - Lincoln. It has been accepted for inclusion in Special Education and Communication Disorders Faculty Publications by an authorized administrator of DigitalCommons@University of Nebraska - Lincoln. 


\title{
Exceptional Children
}

Vol. 73, No. 3, pp. 367-379.

02007 Council for Exceptional Children.

\section{Risk Factors Predictive of the Problem Behavior of Children At Risk for Emotional and Behavioral Disorders}

\author{
J. RON NELSON \\ University of Nebraska, Lincoln \\ SCOTT STAGE \\ University of Washington \\ KRISTIN DUPPONG-HURLEY \\ LORI SYNHORST \\ MICHAEL H. EPSTEIN \\ University of Nebraska, Lincoln
}

ABSTRACT: Logistic regression analyses were used to establish the most robust set of risk factors that would best predict borderlinelclinical levels of problem behavior (i.e., a $\mathrm{t}$ score at or above 60 on the Child Behavior Checklist Total Problem scale) of kindergarten and first-grade children at risk for emotional and behavioral disorders. Results showed that among the 11 risk factor domains considered, 5 were most predictive of borderlinelclinical levels of problem behavior: externalizing behavior pattern, internalizing behavior pattern, early childhood child maladjustment, family functioning, and maternal depression. Within these 5 domains, the most robust set of individual risk factors were difficult child (i.e., temperament, parent management skills, interaction between temperament and parent management skills), destroys own toys, and maternal depression. Results, limitations, future research, and implications are discussed.

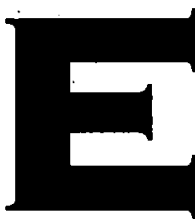

arly problem behavior is highly related to successful school experiences (Gresham, Lane, \& Lambros, 2000; Lyman, 1996). Children who evidence problem behavior at school are likely to struggle early, often, and throughout their school careers (Patterson, 1982; Walker, Colvin, \& Ramsey, 1995).
It is for this reason that practitioners, policy makers, and researchers have become interested in identifying risk factors for emotional and behavioral disorders (E/BD). Indeed, Forness (2003) noted the importance of a developmental psychopathology approach to special education particularly with respect to understanding and treating children with $\mathrm{E} / \mathrm{BD}$. He stated that the 
primary value is in its emphasis on early detection and prevention of disorders, and acknowledging the early trajectories that children establish as they begin to evidence E/BD. Forness suggested that special educators begin to use the science from developmental psychopathology such as information on childhood factors that place children at risk for $\mathrm{E} / \mathrm{BD}$ in designing assessment tools for screening and intervention planning.

The study of risk factors is part of a relatively new discipline of developmental psychopathology that "represents a movement toward comprehending the causes and determinants, course, sequelae, and treatment of childhood disorders" (Cicchetti $\&$ Toth, 1995, p. 373). Risk factors are those variables that when present in a child, increase the likelihood that the child will subsequently evidence E/BD. A risk factor approach is based on the belief that significant exposure to key risk factors is associated with negative, long-term life outcomes (Patterson, Reid, \& Dishion, 1992). Empirical evidence suggests that this process likely operates in the following manner: (a) children and youth are exposed to a host of risk factors over time (e.g., family problems, child neglect/abuse); (b) risk factors are associated with the development of maladaptive behaviors (e.g., restlessness, overactivity, aggression); (c) shortterm outcomes include truancy, peer and teacher rejection, low academic achievement, and school discipline contacts and referrals; and (d) these short-term outcomes, in turn, are predictive of much more serious, long-term outcomes (e.g., $\mathrm{E} / \mathrm{BD}$, school failure, and dropout; Cicchetti \& Nurcombe, 1993).

The risk factor literature can be quite informative in designing assessment tools for screening and intervention planning (Huesmann, Eron, \& Dubow, 2002). Certain physiological and medical factors experienced early in life such as premature birth, low birth weight, and slow neurological development place children at risk for $\mathrm{E} / \mathrm{BD}$ (McCormick, McCarton, Brooks-Gunn, Belt, \& Gross, 1998). Children who demonstrate difficult temperament such as impulsiveness, distractability; irritability, inflexibility, and attention deficit problems are also likely to evidence problem behavior (Brier, 1995). Also, as one would predict children who as infants and toddlers evidence externalizing problem behavior such as aggression, destructiveness, and conduct problems have a high probability of demonstrating problem behavior in school (Gresham et al., 2000). Family factors such as parental mental health and substance abuse histories, marital discord, child maltreatment, and parenting styles are strong predictors of later problem behavior in school (Eckenrode, Rowe, Laird, \& Brathwaite, 1995). None of these risk factors alone is likely to lead to the onset of problem behavior; more than likely it is the presence of several of these variables working together that leads to the development and maintenance of problem behavior. Further, it is likely that there are reciprocal interactions between and among risk factors. For example, a child who has a difficult temperament may not evidence problem behavior if they have parents who have outstanding parent management skills and are not impacted by family stressors (e.g., changes in family composition, maternal depression); whereas, such a child may evidence problem behavior if they have parents who lack parent management skills and are impacted by family stressors.

\section{$A$ child who has a difficult temperament may not evidence problem behavior if they have parents who have outstanding parent management skills and are not impacted by family stressors:}

There are three universally accepted types of risk factors (Kraemer et al., 1997): fixed marker risk factor, variable marker risk factor, and causal risk factor. Fixed marker risk factors cannot be demonstrated to change. For example, belonging to a disadvantaged minority group is a risk factor for low academic achievement (Reynolds, Weissberg, \& Kasprow, 1992), but such membership cannot be changed; therefore, minority status is a fixed marker risk factor. Variable marker risk factors can be demonstrated to change, but when changed, does not necessarily alter the probability of the outcome. For example, a mother's failing to graduate from high school is a risk factor for a child identified as having a disability (Finkelstèin \& Ramey, 1980). However, simply awarding a 
diploma to a mother at the birth of her child ultimately will not change her child's educational trajectory; therefore, maternal possession of a high school diploma is a variable marker risk factor. Causal risk factors can be changed and, when changed, they alter the risk of outcome. For example, high-quality child care for infants has been demonstrated to increase children's academic achievement (Berlin, Brooks-Gunn, McCarton, \& McCormick, 1998); therefore, low-quality child care is a causal risk factor. Causal risk factors include certain child (e.g., cognitive deficits, early behavior and adjustment problems) and family characteristics (e.g., parental psychopathology, poor parenting practices). For example, parent management training has been found to improve the social functioning of children at risk for E/BD (Patterson, 1982).

Although only causal risk factors can be used for intervention planning, all of the risk factors can play a role in the development of assessment tools for screening children at risk for developing $\mathrm{E} / \mathrm{BD}$. The primary purpose of this research was to identify from previously established risk factors for problem behavior (e.g., Greenberg, Lengua, Cole, \& Pinderhughes, 1999; Huffman, Mehlinger, \& Kerivan, 2000; Lowry, Sleet, Duncan, Powell, \& Kolbe, 1995) those variables that would best predict borderline/clinical levels of problem behavior of kindergarten and first-grade children at risk for E/BD. This study builds on the risk factor research in two ways (see Huffman et al. for a review). First, we studied 40 risk factors across 11 domains. Previous research has tended to focus on individual or small numbers of risk factors such as childhood maltreatment (e.g., Kendall-Tackett \& Eckenrode, 1996). Second, we sought to identify the most robust set of risk factors that predict borderline/clinical levels of problem behavior of children at risk for $\mathrm{E} / \mathrm{BD}$ (i.e., a $t$ score at or above 60 on the Child Behavior Checklist, CBCL, Total Problems scale; Achenbach, 2001). Previous research has focused on clinically identified populations (e.g., Walrath et al., 2004). We chose the CBCL because this type of behavior rating scale is typically used by school psychologists in the process of determining students with E/BD (Sattler \& Hoge, 2006).

\section{METHOD}

\section{PARTICIPANTS}

A total of 157 selected kindergarten $(n=78)$ and first-grade $(n=79)$ children at risk for E/BD participated. These children were selected across 3 consecutive school years from seven elementary schools located in a medium-sized city in the Midwest. Parental informed consent was obtained in all cases. Our approved Institutional Review Board procedures did not require that we obtain child assent. These children were recruited to participate in a study of the outcomes of a threetiered behavior prevention model. More specifically, the participants of this study were those children selected to receive a secondary level intervention (i.e., First Step to Success; Walker, Stiller, Golly, Kavanagh, Severson, \& Feil, 1997). A parallel two-step universal screening process was used to identify kindergarten and first-grade participants. The screening process for kindergarten and first-grade participants included the first and second gates of the Early Screening Project (ESP; Walker, Severson, \& Feil, 1995) and Systematic Screening for Behavior Disorders (SSBD; Walker \& Severson, 1990), respectively.

The screening procedure was conducted at the participating schools during the 5th or 6th week of the school year for 3 successive years. At Step 1, kindergarten and first-grade teachers were provided with a definition and examples of externalizing and internalizing behavioral characteristics articulated in the ESP and SSBD, respectively. Teachers then generated two mutually exclusive lists of children. The first list included those children whose characteristic behavior pattern most closely resembled the externalizing behavior description. Teachers then rank ordered these children according to the degree to which their behavior matched the externalizing definition. To generate the second list, an identical procedure was followed to list and rank order children according to the internalizing behavior definition.

At Step 2, kindergarten and first grade teachers completed the three ESP and SSBD scales (i.e., Critical Events Index, Maladaptive Behavior, Adaptive Behavior) on the five highest externalizing and internalizing children identified in Step 1, respectively. The ESP and SSBD Critical Events 
Index has 16 and 33 items (e.g., steals, sets fires), which teachers rank as occurring or not occurring, respectively. The ESP and SSBD Adaptive Behavior scale includes 8 and 12 items that assess teacher- and peer-related adaptive behavior, which teachers rate on a 5-point Likert-type scale, respectively. The ESP and SSBD Maladaptive Behavior scale includes 9 and 11 items that assess teacher- and peer-related problem behavior, which teachers rate on a 5-point Likert-type scale, respectively. Teachers' ratings on the ESP and SSBD Adaptive Behavior and Maladaptive Behavior scales are based on the frequency of children's behavior within the past $30^{\prime}$ days. Test-restest reliabilities for the ESP Critical Events, Adaptive Behavior, and Maladaptive Behavior scales ranged between .75 and .92 across samples of young children (Walker, Severson, et al., 1995). The test-retest reliabilities for the SSBD ranged between .77 and .93 (Walker \& Severson, 1990).

Kindergarten and first-grade children were eligible to participate if they met the respective specified ESP and SSBD criteria. Specifically, kindergarten children with $t$ scores of 60 or more on both the ESP Adaptive Behavior and Maladaptive Behavior scales or with $t$ scores of 70 or more on the ESP Critical Events scale were eligible for participation. First-grade children whose characteristic behavior pattern most closely resembled the externalizing behavior description with $t$ scores of 43 or less and 56 or more on the Adaptive Behavior and Maladaptive Behavior scales, respectively, or $t$ scores of 55 or more on the SSBD Critical Events Index were eligible for participation. First-grade children whose characteristic behavior pattern most closely resembled the internalizing behavior description with $t$ scores of 43 or less and 53 or more on the Adaptive Behavior and Maladaptive Behavior scales, respectively, or $t$ scores of 60 or more on the SSBD Critical Events Index were eligible for participation. Step 3 of the ESP and SSBD were not included because of the significant time and resources required to commit to classroom and playground observations of student behavior, and the reliability of Steps 1 and 2 in identifying children at risk for E/BD (Walker, personal communication, August 15, 2002).

Participant demographic characteristics (i.e., gender, race) and ESP and SSBD Critical Events
Index, Adaptive, and Maladaptive $t$ scores by grade are presented in Table 1. A majority of the participants were boys $(72 \%)$. The race of children was based on the designations provided by guardians. Caregivers of 16 kindergarten and 8 first-grade children designated two categories of race for their child. The overall race breakdown of the children included 119 Caucasians, 28 African Americans, 15 Native Americans, 11 Hispanics/ Latinos, 4 Asian Americans, 2 Hawaiians/Pacific Islanders, and 2 other race. Chi-square analyses with Yates correction on these nominal data showed no effects for grade: gender, $\chi^{2}(1)=$ $0.167, p=.628$, and race, $\chi^{2}(6)=3.98, p=.681$.

\section{DEPENDENT MEASURES}

Problem Behavior. The CBCL (Achenbach, 2001) Total Problems broad band scale was used to assess the problem behavior of children. The parent/guardian rates the child on each item indicating the severity of the problem on a scale of 0 (no problem) to 2 (severe problem). The $\mathrm{CBCL}$ provides a total scale score (i.e., Total Problems), two broad band scale scores (i.e., Internalizing, Externalizing), and six narrow band subscale scores (i.e., Affective Problems, Somatic Problems, Attention Deficit/Hyperactivity Problems, Oppositional Defiant Problems, Conduct Problems). The Total Problems scale scores were converted to dichotomous "problem behavior absent" (i.e., a $t$ score at or above 60 on the Total Problem scale) or "problem behavior present" (i.e., a $t$ score below 60 on the Total Problem scale) using the established CBCL interpretive framework. The CBCL test-retest and internal consistency values for the Total Problems, Externalizing, and Internalizing broad band scales ranged from .72 to .95 and .65 to .92 , respectively (Achenbach).

Risk Factors. The risk factor profile measures included a structured developmental interview, a standardized measure designed to assess family functioning, and a measure to assess maternal depression. The family functioning domain comprised three risk factors, whereas the maternal depression comprised a single risk factor. The standardized measures were dichotomized into risk absent (0) or risk present (1) according to the following specified criteria to provide a consistent 
TABLE 1

Demographic Characteristics and ESP and SSBD Scores of Kindergarten and First-Grade Children

\begin{tabular}{|c|c|c|c|c|c|c|c|c|}
\hline \multirow[b]{2}{*}{ Demographic variable } & \multicolumn{4}{|c|}{$\begin{array}{c}\text { Kindergarten } \\
(\mathrm{N}=78)\end{array}$} & \multicolumn{4}{|c|}{$\begin{array}{c}\text { First Grade } \\
(\mathrm{N}=79)\end{array}$} \\
\hline & $\mathrm{n}$ & $\%$ & M & SD & $\mathrm{n}$ & $\%$ & M & SD \\
\hline \multicolumn{9}{|l|}{ Gender } \\
\hline Male & 55 & 70 & & & 58 & 73 & & \\
\hline Female & 23 & 39 & & & 21 & 27 & & \\
\hline \multicolumn{9}{|l|}{ Race $^{\mathbf{a}}$} \\
\hline African American & 17 & 22 & & & 11 & 14 & & \\
\hline Asian & 3 & 4 & & & 1 & 1 & & \\
\hline Hawaiian/Pacific Island & 1 & 1 & & & 1 & 1 & & \\
\hline Hispanic/Latino & 5 & 6 & & & 6 & 8 & & \\
\hline Native American & 10 & 13 & & & 5 & 6 & & \\
\hline Caucasian & 57 & 73 & & & 62 & 80 & & \\
\hline Other Race & 1 & 1 & & & 1 & 1 & & \\
\hline \multicolumn{9}{|l|}{ ESP/SSBD Scores } \\
\hline Critical Events Index & & & 55.9 & 8.6 & & & 60.5 & 9.9 \\
\hline Maladaptive Behavior & & & 74.2 & 7.3 & & & 61.0 & 7.3 \\
\hline Adaptive Behavior & & & 70.1 & 7.2 & & & 40.2 & 9.0 \\
\hline
\end{tabular}

Note. ESP = Early Screening Project. SSBD = Systematic Screening for Behavior Disorders.

${ }^{\mathrm{a}}$ Caregivers of 16 kindergarten and 8 first-grade children designated two categories of race for their child.

Percentages are based on the overall self-reports of race.

response format across all of the risk factors. Descriptions of the risk factor measures follow.

- Structured Developmental Interview. A Structured Developmental Face-to-Face Interview (Nelson \& Epstein, 2002) was designed to assess 36 child developmental risk factors across nine domains (described below). A dichotomous response format was used by parents to indicate the presence (i.e., yes, no) of each risk factor. Demographic information collected included (a) gender of child; (b) age of the mother, father, and child; (c) self-designated race of the biological mother, father, and child (i.e., African American, Asian, Hispanic/Latino, Native Hawaiian/Pacific Islander, Caucasian, Native American, other); (d) primary language spoken at home (i.e., English, Spanish, other); and (e) current primary caregiver of the child and relationship to child (i.e., birth mother, birth father, legal guardian, other). The psychometric characteristics of the Structured Developmental Face-to-Face Interview were not assessed.

Although each of the 36 child development risk factor items was designed to measure a different risk factor, conceptually similar items were grouped into nine broad risk factor domains. The domains and associated risk factors include the following:

- Prenatal (i.e., maternal emotional distress, maternal medical problems).

- Natal (i.e., premature, unusual delivery).

- Postnatal (i.e., medical problems such as breathing problems, umbilical cord around neck, blue color, yellow color; prolonged hospital stay).

- Externalizing behavior pattern during early childhood (i.e., overactive, impulsive, stubborn, temper outbursts, aggressive, destroyed toys, fearless). 
- Internalizing behavior pattern during early childhood (i.e., shy or timid, fearful, preferred to be alone, socially withdrawn, cautious, difficulty sleeping).

- Childhood maladjustment (i.e., psychiatric hospitalization, runaway, physically abusive to others, abusive to animals).

- Childhood maltreatment (i.e., sexually abused, physically abused).

- Antisocial and psychiatric family history (i.e., domestic violence, mental illness, psychiatric hospitalization, substance abuse, substance abuse treatment, convicted of a crime).

- Family structure (i.e., one parent, no high school diploma) and socioeconomic status (i.e., free or reduced-price lunch).

- Family Functioning. The Parenting Stress Index/Short Form (PSI/SF; Abidin, 1995) was used to measure family functioning. The PSI/SF indicates the degree of stress an individual is experiencing due to parenting. The $\mathrm{PSI} / \mathrm{SF}$ is a direct derivative of the Parenting Stress Index full-length test (Abidin). The PSI/SF has three subscales: Parental Distress, Parent-Child Dysfunctional Interaction, and Difficult Child. Each of the subscales comprised 12 items. The Parental Distress score reflects stresses derived from (a) an impaired sense of parenting competence, (b) restrictions placed on other life roles, (c) conflict with the child's other parent, and (d) lack of social support. The Parental Distress score also reflects the presence of parental depression. The Parent- Child Dysfunctional Interaction score indicates the parent's interactions with their child are not reinforcing and that the child does not meet their expectations. The Difficult Child score reflects the behavioral characteristics of the child that influence parental management. Respondents marked each item on a 5-point Likerttype scale: strongly agree, agree, not sure, disagree, and strongly disagree. The Parental Distress, Parent-Child Dysfunctional Interaction, and Difficult Child subscales yield percentile scores. The normal range for scores is within the 15 th to 85 th percentiles. The percentile scale scores were converted to dichotomous risk absent or risk present values (i.e., $\leq 85$ and $>85$ ) for all analyses. The coefficient alpha and test-retest reliabilities for the PSI/SF composite and Parental Distress, Parent-Child Dysfunctional Interaction, and Difficult Child subscales range from .80 to .91 and .68 to .85 , respectively (Abidin).

- Maternal Depression. The Beck Depression Inventory Second Edition (BDI-II; Beck, Steer, \& Brown, 1996) was used to measure the severity of the maternal depression experienced by the child's mother. The BDI-II includes 21 items that assess symptoms of depression corresponding to criteria for diagnosing depressive disorders listed in the $D i$ agnostic and Statistical Manual of Mental Disorders (4th ed.; DSM-IV, American Psychiatric Association, 1994). Mothers respond to the 21 items on a 4-point Likert-type scale ranging from 0 to 3 corresponding to a series of statement. For example, for the first item (i.e., sadness) the scale includes: 0 ( $I$ do not feel sad), 1 (I feel sad much of the time), 2 (I am sad all the time), and 3 (I am so sad or unhappy that I can't stand it). The BDI-II is scored by summing the ratings for the 21 items. The total score is broken down into four interpretive categories: $0-13$ : minimal depression, 14-19: mild depression, 20-28 moderate depression, and 29-63: severe depression. Based on the recommended clinical interpretation (Beck et al.), the total score was converted to dichotomous risk absent or risk present values (i.e., $<20$ and $\geq 20$ ) for all analyses. The coefficient alpha and test-retest reliability for the BDI-II was .86 and .93, respectively (Beck et al.).

\section{Data Collection Procedures}

Training. Research staff consisted of individuals hired specifically to collect data on a number of ongoing research projects. Staff participated in a total of $50 \mathrm{hr}$ of supervised training and practice to administer the measures as well as child outcome measures used in our study of a three-tiered behavior prevention model. The training process 
included the following: (a) trainer provided an overview of each measure and associated assessment protocol; (b) trainer modeled and practiced each assessment protocol with staff; (c) simulated practice conditions with structured feedback were conducted to ensure that staff obtained a high level of skill performance with each assessment protocol; (d) staff were observed (via a one-way mirror) administering each assessment protocol under simulated conditions and were required to achieve a high degree of proficiency (i.e., $\geq 90 \%$ accuracy); (e) staff observed an experienced member administer each assessment protocol in the field; (f) an experienced data collector monitored staff's initial administrations of each assessment protocol in the field and provided structured feedback on their proficiency (i.e., $\geq 90 \%$ accuracy); (g) staff were observed two times administering each assessment protocol under field conditions and were required to achieve a high degree of proficiency (i.e., $\geq 90 \%$ ); and (h) staff were observed (unannounced) administering each of the assessment protocols in the field. Staff who failed to meet the proficiency standards during simulated or field conditions received additional training and practice until they achieved the criterion.

\section{Staff participated in a total of $50 \mathrm{hr}$ of supervised training and practice to administer the measures as well as child outcome measures used in our study of a three-tiered behavior prevention model.}

Data Collection. The primary caregiver for each child was assessed individually either in their homes or a private location in their child's school in one 30- to 45-min session. Unless there were safety concerns (e.g., significant mental health problems), one staff member administered the Structured Developmental Face-to-Face Interview, PSI/SF, and BDI-II protocols. Two staff members administered the protocols in those cases in which there were safety concerns. The staff members spent several minutes establishing rapport with the primary caregiver prior to administering the assessment protocols. The Struc- tured Developmental Face-to-Face Interview was always administered first. The PSI/SF, BDI-II, and $C B C L$ were then administered in a counterbalanced fashion.

\section{RES U LTS}

\section{STATISTICAL ANALYSIS}

Two logistic regression analysis procedures were used to determine the most reliable and robust prediction of borderline/clinical levels of total problem behavior using the risk factors. The target variables for each of the logistic regression analysis procedures was the dichotomized (i.e., problem behavior absent, $n=79$, problem behavior present, $n=76$ ) CBCL Total Problems broad band scores. The purpose of the initial logistic regression analysis procedure was to identify the most salient set of domains and represented risk factor variables within each domain to include in the final stepwise logistic regression model. Demographic characteristics (i.e., gender, ethnicity, disability) and each of the 36 risk factor variables were entered as a block of variables by domain into a logistic regression analysis that predicted total problem behavior. Prior to the initial analysis, logistic regression analyses by domain were conducted to detect outliers in the distribution that might overly influence the results of the analysis. In each analysis, there were no examples of outliers greater than a $\mathrm{z}$ score of two; therefore no corrections were made for outliers in additional analyses. In addition, because multiple independent variables were entered into each analysis, multicollinearity was assessed for each analysis separately. For each analysis, a dummy variable was created for each variable except one, which makes it the reference category, and entered it into a linear regression analysis. The collinearity diagnostics were evaluated. In particular, the Variance Inflation Factor (VIF; i.e., $1 / 1-R^{2}$ for all remaining independent variables) for each variable was tested. VIF scores above 2.5 are cause for concern (Allison, 1999). None of the variables in any of the analyses approached a VIF score of 2.5. Therefore, none of the initial logistic regression analyses by domain found violations that were due to outliers in the distribution or that were due to artifacts caused by multicollinearity. 
In order to be considered for the second stepwise logistic regression analysis, an omnibus $\chi^{2}$ statistic needed to be statistically significant $(p<$ $.05)$ for each at-risk domain that was entered as a block (e.g., in the prenatal domain both medical and emotional pregnancy problems were tested together). Only the individual risk factor variables within domains that were statistically significant $(p<.05)$ predictors of total problem behavior (i.e., present, absent) were considered for the final stepwise logistic regression analysis procedure. In addition, diagnostics tests to detect outliers and multicollinearity were performed for the second logistic regression analysis as described above. No violations were detected. The purpose of the final stepwise logistic regression procedure was to identify the risk factor variables that provided the most reliable and robust prediction of total problem behavior.

\section{INITIAL LOGISTIC REGRESSION TO IDENTIFY STATISTICALLY SIGNIFICANT PREDICTORS}

The results of the initial regression analysis procedure are presented in Table 2 . A statistically significant omnibus $\chi^{2}$ statistic was obtained for the externalizing, $\chi^{2}(7)=26.47, p=.000$; internalizing, $\chi^{2}(6)=15.67, p=.016$; child maladjustment, $\chi^{2}(4)=29.04, p=.000$; family functioning, $\chi^{2}(3)=37.58, p=.000$; and maternal depression, $\chi^{2}(1)=14.47, p=.000$ risk factor domains. The omnibus $\chi^{2}$ statistic for the remaining risk factor domains was not statistically significant.

Total problem behavior was statistically predicted by children who (a) destroyed their own toys $(B=1.30, S E=.47$, Wald $=7.92, p=.005$; Odds Ratio $=3.72: 1$ ); (b) had difficulty sleeping $(B=1.05, S E=.48$, Wald $=4.79, p=.029$; Odds Ratio $=2.90: 1)$; $(c)$ were physically abusive to others $(B=1.77, S E=.50$, Wald $=12.5, p=.000$; Odds Ratio $=5.80: 1)$; and (d) were difficult to parent $(B=1.93, S E=.45$, Wald $=18.2, p=$ .000 ; Odds Ratio $=7.00: 1)$. Total problem behavior was also statistically predicted by maternal depression $(B=2.81, S E=1.05$, Wald $=7.18, p=$ .000 ; Odds Ration $=16 \cdot 6: 1)$. Although gender $(B$ $=-0.94, S E=3.83$, Wald $=6.06, p=.014$; Odds Ration $=1.39: 1)$ and postnatal medical problems
$(B=0.74, S E=3.61$, Wald $=4.16, p=.041$; Odds Ration $=2.09: 1)$ were statistically significant $(p<.05)$, they were not included in further analyses because the omnibus $\chi^{2}$ statistic for the demographic (gender) and postnatal (maternal medical problems) domains was not statistically significant $(p>.05)$.

\section{STEPWISE LOGISTIC REGRESSION USING STATISTICALLY SIGNIFICANT PREDICTORS}

A stepwise forward conditional logistic regression was conducted to predict total problem behavior with the five statistically significant risk factors identified in the initial logistic regression analysis (i.e., destroys own toys, sleep difficulty, physically abusive to others, difficult to parent, maternal depression). Step 1 difficult child entered with $R^{2}=$ .27. Step 2 destroys own toys entered with $R^{2}=$ .33 , and in Step 3, the final step, maternal depression entered with $R^{2}=.38$. The overall correct classification of cases was $71.5 \%$ with true negatives correctly classified $71 \%$ of the time and true positives correctly classified $72 \%$ of the time. The results of the final step are presented in Table 3. The exponential coefficients in the far right column of Table 3 are equivalent to the prediction or odds that the variable predicts the relationship with the outcome measure. The three largest predictors of total problem behavior were children who destroyed their own toys (Odds Ratio = 3.95:1), were difficult to parent (Odds Ratio $=$ 6.17:1), and had mothers who were depressed (Odds Ratio $=10.48: 1)$. Because stepwise regression results may provide results that are influenced by the statistical procedure that selects the entrance of the variable into the equation, four other logistical regressions were conducted using different algorithms for entry into the final results: simultaneous entry of all the variables, forward likelihood ratio, backward conditional entry, and backward likelihood ratio. All the results yielded similar results to those reported above. Odds ratios for the different analyses for destroyed their own toys ranged from 3.19-3.95 to 1, for difficult to parent from 5.0-6.17 to 1 , and for maternal depression $10.48-11.74$ to 1 . Results showed that in forward conditional, forward likelihood ratio, backward conditional, and backward likelihood ratio that the three same variables were 
TABLE 2

Initial Logistic Regression Predicting Total Problem Behavior With At-Risk Domains and Represented Risk Factors Within Domains

\begin{tabular}{|c|c|c|c|c|c|c|}
\hline Domain & Omnibus $\chi^{2}$ & Risk Factor & B & SE & Wald Statistic & Odds Ratio \\
\hline Demographic & $x^{2}(3)=6.90, p=.075$ & $\begin{array}{l}\text { Gender } \\
\text { Ethnicity } \\
\text { Disability }\end{array}$ & $\begin{array}{r}-0.94 \\
0.21 \\
0.22\end{array}$ & $\begin{array}{l}.383 \\
.386 \\
.519\end{array}$ & $\begin{array}{l}6.06, p=.014 \\
0.31, p=.580 \\
0.23, p=.880\end{array}$ & $\begin{array}{l}0.39: 1 \\
1.24: 1 \\
0.93: 1\end{array}$ \\
\hline Prenatal & $\chi^{2}(2)=3.80, p=.149$ & $\begin{array}{l}\text { Emotional distress } \\
\text { Medical problems }\end{array}$ & $\begin{array}{r}-0.39 \\
0.76\end{array}$ & $\begin{array}{l}.408 \\
.401\end{array}$ & $\begin{array}{l}0.92, p=.335 \\
3.57, p=.059\end{array}$ & $\begin{array}{l}0.68: 1 \\
2.13: 1\end{array}$ \\
\hline Natal & $\chi^{2}(2)=0.56, p=.756$ & $\begin{array}{l}\text { Premature } \\
\text { Unusual delivery }\end{array}$ & $\begin{array}{l}0.17 \\
0.19\end{array}$ & $\begin{array}{l}.448 \\
.320\end{array}$ & $\begin{array}{l}0.15, p=.693 \\
0.36, p=.548\end{array}$ & $\begin{array}{l}1.19: 1 \\
1.20: 1\end{array}$ \\
\hline Postnatal & $\chi^{2}(2)=8.07, p=.087$ & $\begin{array}{l}\text { Medical problems } \\
\text { Prolonged stay }\end{array}$ & $\begin{array}{r}0.74 \\
-0.24\end{array}$ & $\begin{array}{l}.361 \\
.568\end{array}$ & $\begin{array}{l}4.16, p=.041 \\
0.01, p=.967\end{array}$ & $\begin{array}{l}2.09: 1 \\
0.09: 1\end{array}$ \\
\hline $\begin{array}{l}\text { Externalizing } \\
\text { behavior }\end{array}$ & $\chi^{2}(7)=26.47, p=.000$ & $\begin{array}{l}\text { Overactive } \\
\text { Impulsive } \\
\text { Stubborn } \\
\text { Temper outbursts } \\
\text { Aggressive } \\
\text { Destroyed toys } \\
\text { Fearless }\end{array}$ & $\begin{array}{r}0.53 \\
0.53 \\
-0.39 \\
0.34 \\
0.23 \\
1.30 \\
-0.05\end{array}$ & $\begin{array}{l}.386 \\
.385 \\
.441 \\
.405 \\
.428 \\
.470 \\
.385\end{array}$ & $\begin{array}{l}1.86, p=.173 \\
1.91, p=.167 \\
0.80, p=.371 \\
0.72, p=.396 \\
0.28, p=.595 \\
7.92, p=.005 \\
0.02, p=.897\end{array}$ & $\begin{array}{l}1.70: 1 \\
1.70: 1 \\
0.67: 1 \\
1.14: 1 \\
1.30: 1 \\
3.72: 1 \\
0.95: 1\end{array}$ \\
\hline $\begin{array}{l}\text { Internalizing } \\
\text { behavior }\end{array}$ & $x^{2}(6)=15.67, p=.016$ & $\begin{array}{l}\text { Shy or timid } \\
\text { Fearful } \\
\text { Prefers to be alone } \\
\text { Socially withdrawn } \\
\text { Cautious } \\
\text { Difficulty sleeping }\end{array}$ & $\begin{array}{l}0.39 \\
0.63 \\
0.57 \\
0.34 \\
0.15 \\
1.05\end{array}$ & $\begin{array}{l}.386 \\
.502 \\
.522 \\
.601 \\
.361 \\
.480\end{array}$ & $\begin{array}{l}0.99, p=.318 \\
1.60, p=.206 \\
1.18, p=.592 \\
0.32, p=.571 \\
0.18, p=.671 \\
4.79, p=.029\end{array}$ & $\begin{array}{l}1.47: 1 \\
1.88: 1 \\
1.76: 1 \\
1.40: 1 \\
1.16: 1 \\
2.90: 1\end{array}$ \\
\hline $\begin{array}{l}\text { Childhood } \\
\text { maladjustment }\end{array}$ & $\chi^{2}(4)=29.04, p=.000$ & $\begin{array}{l}\text { Psychiatric hospital } \\
\text { Runaway } \\
\text { Physically abusive } \\
\text { Abusive to animals }\end{array}$ & $\begin{array}{r}-1.12 \\
7.05 \\
1.77 \\
0.15\end{array}$ & $\begin{array}{r}.441 \\
18.650 \\
.500 \\
.361\end{array}$ & $\begin{aligned} 0.01, p & =.978 \\
0.14, p & =.706 \\
12.50, p & =.000 \\
0.18, p & =.120\end{aligned}$ & $\begin{array}{r}0.33: 1 \\
1150: 1 \\
5.80: 1 \\
1.16: 1\end{array}$ \\
\hline $\begin{array}{l}\text { Childhood } \\
\text { maltreatment }\end{array}$ & $x^{2}(2)=3.23, p=.199$ & $\begin{array}{l}\text { Sexually abused } \\
\text { Physically abused }\end{array}$ & $\begin{array}{r}1.39 \\
-0.19\end{array}$ & $\begin{array}{l}.870 \\
.870\end{array}$ & $\begin{array}{l}2.58, p=.108 \\
0.48, p=.827\end{array}$ & $\begin{array}{l}4.00: 1 \\
0.83: 1\end{array}$ \\
\hline Family history & $\chi^{2}(6)=12.03, p=.061$ & $\begin{array}{l}\text { Domestic violence } \\
\text { Mental illness } \\
\text { Psychiatric hospital } \\
\text { Substance abuse } \\
\text { Substance abuse } \\
\text { treatment } \\
\text { Convicted }\end{array}$ & $\begin{array}{r}0.28 \\
0.43 \\
0.34 \\
0.73 \\
\\
0.23 \\
-0.39\end{array}$ & $\begin{array}{l}.370 \\
.467 \\
.564 \\
.450 \\
\\
.490 \\
.420\end{array}$ & $\begin{array}{l}0.59, p=.442 \\
0.85, p=.358 \\
0.36, p=.546 \\
2.64, p=.104 \\
\\
0.21, p=.646 \\
0.89, p=.358\end{array}$ & $\begin{array}{l}1.30: 1 \\
1.50: 1 \\
1.40: 1 \\
2.00: 1 \\
\\
1.30: 1 \\
0.67: 1\end{array}$ \\
\hline $\begin{array}{l}\text { Family } \\
\text { structure }\end{array}$ & $\chi^{2}(3)=6.77, p=.080$ & $\begin{array}{l}\text { One parent } \\
\text { No high school } \\
\text { diploma } \\
\text { Free or reduced- } \\
\text { price lunch }\end{array}$ & $\begin{array}{r}0.62 \\
-0.31\end{array}$ & .411 & $\begin{array}{l}2.30, p=.129 \\
0.37, p=.545 \\
2.71, p=.099\end{array}$ & $\begin{array}{l}1.90: 1 \\
0.70: 1\end{array}$ \\
\hline $\begin{array}{l}\text { Family } \\
\text { functioning }\end{array}$ & $\chi^{2}(3)=37.58, p=.000$ & $\begin{array}{l}\text { Parental distress } \\
\text { Parent-child } \\
\text { dysfunction } \\
\text { Difficult child }\end{array}$ & $\begin{array}{l}0.48 \\
1.93\end{array}$ & $\begin{array}{l}.388 \\
.450\end{array}$ & $\begin{aligned} 0.17, p & =.897 \\
2.71, p & =.265 \\
18.20, p & =.000\end{aligned}$ & $\begin{array}{l}1.90: 1 \\
7.00: 1\end{array}$ \\
\hline $\begin{array}{l}\text { Maternal } \\
\text { depression }\end{array}$ & $x^{2}(1)=14.47, p=.000$ & Maternal depression & 2.81 & 1.050 & $7.18, p=.007$ & $16.60: 1$ \\
\hline
\end{tabular}


TABLE 3

Stepwise Logistic Regression Predicting Total Problem Behavior With Statistically Significant At-Risk Factors

\begin{tabular}{llccc}
\hline Total Problem Behavior & \multicolumn{1}{c}{ Variables } & B & SE & Exp $(\mathrm{B})$ \\
\hline Final step & Destroys own toys & $1.373, p=.002$ & .454 & 3.95 \\
& Difficult child & $1.909, p=.000$ & .425 & 6.17 \\
& Maternal depression & $2.349, p=.035$ & 1.117 & 10.48 \\
\hline
\end{tabular}

Note. Variables not in the equation for the Total Problems were sleep difficulty and physically abusive to others. Exp $=$ exponential coefficient.

identified as contributing significant changes to the model if they were removed at $p<.01$.

\section{DISCUSSION}

There are calls by special educators to use a developmental psychopathology approach to better understand and treat children with E/BD (Forness, 2003). Such an approach was applied in this study to identify those variables that would best predict borderline/clinical levels of problem behavior of kindergarten and first-grade children at risk for $\mathrm{E} / \mathrm{BD}$. Based on the data analyses there were two primary findings. First, among the 11 risk factor domains studied, 5 were most predictive of problem behavior including externalizing; internalizing, child maladjustment, family functioning, and maternal depression. Second, when these domains and the individual variables they represent were further analyzed via a stepwise logistic regression, the best predictors of problem behavior were difficult child, destroys own toys, and maternal depression. Indeed, using these 3 risk factors led to correctly classifying more than $70 \%$ of the sample as with or without problem behavior.

The difficult child risk factor focuses on the child's temperament; the parents' overall behavior management skills and the interaction of the two (Abidin, 1995). The difficult child predictor suggests that in cases in which the child is demanding, moody, easy to upset, or stubborn and the parents are inept in setting limits or controlling the environmental contingencies, the child is at risk for $E / B D$. Parents who reported that their children were difficult to manage were more than six times more likely to evidence problem behavior. This finding was not unexpected as previous research identified temperament (Brier, 1995) and parenting practices (Huesmann et al., 2002; Patterson, 1982) as risk factors. Destroys own toys risk factor is a significant observation by the parent/caregiver that the child is capable of significant problem behavior. Destroying one's possessions is very likely associated with other behaviors such as aggression, peer problems, and noncompliance. Children whose parents reported this behavior were almost four times more likely to have behavior problems. Other researchers have noted that behaviors'such as destroys own toys are highly predictive of later school problems (Walker, Severson, et al., 1995). The maternal depression risk factor is a well-documented variable associated with behavior problems in young children (Papp, 2004). Very likely depressed mothers because of their own pathology find the task of parenting to be overwhelming and fail to evidence effective parenting skills while serving as poor models for their children. In the present study, children of mothers who reported that they were depressed were over 10 times more likely to have behavior problems. Although the difficult child combines a fixed risk factor (temperament) and a causal risk factor. (parenting practices), destroys own toys (externalizing adjustment problems) and maternal depression (parental psychopathology) are both causal factors. The causal factors that can be altered and when changed may reduce the risk of the child evidencing problem behavior could be used for intervention planning. The fixed and causal risk factors could be used to develop assessment tools for screening.

\section{LIMITATIONS}

Similar to most educational research, the present study has several limitations. Perhaps the most 
significant limitation is the location of the sample under study. The children and families in the study were selected from seven elementary schools in a mid-sized Midwestern city. Thus, the racial, ethnic, and socioeconomic demographic backgrounds of the sample limit the statements that can be generalized to children in other settings. Obviously, the present study needs to be replicated with other, more diversified samples. Second, teacher and parent/caregiver reports of child behavior and parent/caregiver reports of family functioning were the sole source of data in the present study. As such, the data were restricted to adult perceptions of past and present functioning. It is recommended that in future research attempts are made to validate past adult recollections through archival record reviews and to collect direct observation measures of parent-child and teacher-child interactions in home and school settings. Third, related to this issue, the psychometric characteristics of the Structured Developmental Face-to-Face Interview were not assessed. Thus, it is unclear how stable parent/caregiver's reports of family functioning were in the present study. Fourth, obviously the types of at-risk variables that were included and the data collection measures we selected contributed to the findings. It may be that if other atrisk variables and measures were included, then the data reported on these children and families may have been different.

\section{Parents who reported that their children were difficult to manage were more than six times more likely to evidence problem behavior.}

\section{FUTURE RESEARCH}

The findings and limitations of the present study suggest issues that need to be addressed through future research efforts. First, as already stated, the present study needs to be replicated with a larger more diverse sample that includes urban and rural samples and children and families from different ethnic and socioeconomic backgrounds. Also, these replications need to include a broader array of measures such as direct measures of social and academic functioning. Second, the research focused on which risk factors predicted behavior problems at the beginning of the school year. Equally important is to identify the variables that predict problem behavior during and at the conclusion of the school year. Third, all of the risk factor data were provided by the parents/caregivers for kindergarten and first-grade students. The research should be extended downward to 4and 5-year-old children enrolled in preschool classrooms to determine if the present results can be replicated with a younger population. Fourth, there is a need to identify those risk factors that are predictive of academic difficulties because it is hypothesized that problem behavior and academic difficulties may emerge from the same etiological and environmental risk factors such as parent-child interactions (Huffman et al., 2000). Finally, a similar line of research should be conducted to identify the most robust set of protective factors that increase the resilience of children at risk for $\mathrm{E} / \mathrm{BD}$. A clear understanding of the characteristics, variables, and/or conditions present in individuals that enhance their resiliency and increase resistance to risk for the development of E/BD would serve to advance assessment and intervention procedures.

\section{IMPLICATIONS}

A clear message of the present study is that there are some risk factors that are reasonably accurate predictors of parent/caregiver-reported social behavior of young children. Indeed, in the present study, among a standard list of 40 potential risk factors, 3 factors (i.e., destroys own toys, difficult child, maternal depression) were found to be the most accurate predictors of problem behavior. This suggests that school personnel and developers of early screening tools for children at risk for E/BD consider including items that address these variables. Specifically, comprehensive screening programs to identify young children at risk for such disorders should include items for parents and caregivers to report on the child's play with toys, early negative parent-child interactions, and maternal depression. The results of the present study suggest that these items will be highly predictive of significant child problem behavior. 


\section{REFERENCES}

Abidin, R. E. (1995). Parenting Stress Index/Short Form (PSI/SF). Lutz, FL: Psychological Assessment Resources.

Achenbach, T. M. (2001). Manual for the child behavior checklist/4-18 and 2001 profile. Burlington: University of Vermont, Department of Psychiatry.

Allison, P. D. (1999). Logistic repression using the SAS system: Theory and application. Cary, NC: SAS Institute.

American Psychiatric Association. (1994). Diagnostic and statistical manual of mental disorders (4th ed.). Washington, DC: Author.

Beck, A. T., Steer, R. A., \& Brown, G. K. (1996). Beck Depression Inventory-II (BDI-II). San Antonio, TX: Harcort.

Berlin, L. J., Brooks-Gunn, J., McCarton, C., \& McCormick, M. C. (1998). The effectiveness of early intervention: Examining risk factors and pathways to enhance development. Preventive Medicine, 27, 238-245.

Brier, N. (1995). Predicting antisocial behavior in youngsters displaying poor academic achievement: A review of risk factors. Journal of Developmental and Behavioral Pediatrics, 16, 271-276.

Cicchetti, D., \& Nurcombe, B. (Eds.). (1993). Toward a developmental perspective on conduct disorder [Special issue]. Development and Psychopathology, 5(1/2).

Cicchetti, D., \& Toth, S. (1995). Developmental psychopathology and disorders of affect. In D. Cicchetti \& D. Cohen (Eds.), Developmental psychopathology: Vol. 2. Risk, disorder, and adaptation (pp. 369-420). New York: Wiley.

Eckenrode, J., Rowe, E., Laird, M., \& Brathwaite, J. (1995). Mobility as a mediator of the effects of child maltreatment on academic performance. Child Development, 66, 1130-1142.

Finkelstein, N. W., \& Ramey, C. T. (1980). Information from birth certificates as a risk index for educational handicap. American Journal of Mental Deficiency, $84,546-552$.

Forness, S. R. (2003). Parting reflections on education of children with emotional or behavioral disorders. $B e$ havioral Disorders, 28, 198-201.

Greenberg, M., Lengua, L., Cole, J., \& Pinderhughes, E. (1999). Predicting developmental outcomes at school entry using a multiple-risk model: Four American communities. Developmental Psychology, 35, 403-417.

Gresham, F. M., Lane, K. L., \& Lambros, K. M. (2000). Comorbidity of conduct problems and
ADHD: Identification of "fledgling psychopaths." Journal of Emotional \& Behavioral Disorders, 8, 83-93.

Huesmann, L. R., Eron, L. D., \& Dubow, E. F. (2002). Childhood predictors of adult criminality: Are all risk factors reflected in childhood aggressiveness? Criminal Behavior and Mental Health, 12, 185-208.

Huffman, L. C., Mehlinger, S. L., \& Kerivan, A. S. (2000). Risk factors for academic and behavioral problem at the beginning of school. Retrieved March 2006, from http://www.ce-credit.com/articles/ riskfactorsacademic.pdf

Kendall-Tackett, K. A., \& Eckenrode, J. (1996). The effects of neglect on academic achievement and disciplinary problems: A developmental perspective. Child Abuse and Neglect, 20, 161-169.

Kraemer, H. C., Kazdin, A. E., Offord, D. R., Kessler, R. C., Jensen, P. S., \& Kupfer, D. J. (1997). Coming to terms with the terms of risk. Archives of General Psychiatry, 54, 337-343.

Lowry, R., Sleet, D., Duncan, C., Powell, K., \& Kolbe, L. (1995). Adolescents at risk for violence. Educational Psychology Review, 7, 7-39.

Lyman, D. R. (1996). Early identification of chronic offenders: Who is a fledgling psychopath? Psychological Bulletin, 120, 209-234.

McCormick, M. C., McCarton, C., Brooks-Gunn, J., Belt, P., \& Gross, R. T. (1998). The infant health and development program: Interim summary. Journal of Developmental and Behavioral Pediatrics, 19, 359-370.

Nelson, J. R., \& Epstein, M. H. (2002). Risk Factor Developmental Intake Inventory. Lincoln: University of Nebraska.

Papp, L. M. (2004). Pathways among marital distress, parental symptomatology, and child adjustment. Journal of Marriage and Family, 66, 110-128.

Patterson, G., Reid, J., \& Dishion, T. (1992). Antisocial boys. Eugene, OR: Castalia.

Patterson, G. R. (1982). Coercive family process. Eugene, OR: Castalia.

Reynolds, A. J., Weissberg, R. P., \& Kasprow, W. J. (1992). Prediction of early social and academic adjustment of children from the inner city. Journal of Community Psychology, 20, 599-624.

Sattler, J. M., \& Hoge, R. D. (2006). Assessment of children: Behavioral, social, and clinical foundations (5th ed.). La Mesa, CA: Sattler.

Walker, H., \& Severson, H. (1990). Systematic Screening for Behavior Disorders (SSBD). Longmont, CO: Sopris West. 
Walker, H., Severson, H., \& Feil, E. G. (1995). Early Screening Project (ESP). Longmont, CO: Sopris West.

Walker, H., Stiller, B., Golly, A., Kavanagh, K., Severson, H., \& Feil, E. (1997). First step to success: Helping young children overcome antisocial behavior (an early intervention program for grades $\mathrm{K}-3$ ). Longmont, $\mathrm{CO}$ : Sopris West.

Walker, H. M., Colvin, G., \& Ramsey, E. (1995). Antisocial behavior in school: Strategies and best practices. $\mathrm{Pa}$ cific Grove, CA: Brooks/Cole.

Walrath, C. M., Petras, H., Mandell, D. S., Stephens, R. L., Holden, E. W., \& Leaf, P. J. (2004). Gender differences in patterns of risk factors among children receiving mental health services: Latent class analyses. Journal of Behavioral Health Services \& Research, 31, 297-311.

\section{ABOUT THE AUTHORS}

J. RON NELSON (CEC NE Federation), Associate Professor, University of Nebraska, Lincoln.

scott STAGE (CEC WA Federation), Associate Professor, University of Washington, Seattle.
KRISTIN DUPPONG-HURLEY (CEC NE Federation), Assistant Research Professor; LORI SYNhorst (CEC NE Federation), Assistant Research Professor; and michael h. EPSTEIN (CEC NE Federation), Professor, University of Nebraska, Lincoln.

Correspondence concerning this article should be addressed to J. Ron Nelson, Center for At-Risk Children's Services, 202 Barkley Center, Lincoln, NE 68583-0732. Phone: 402-472-0283; Fax: 402-472-7697 (e-mail: rnelson8@unl.edu).

Preparation of this manuscript was supported in part by a grant from the U.S. Department of Education, Office of Special Education Programs (No. H324X010010). Opinions expressed do not necessarily reflect the position of the U.S. Department of Education, and no endorsement should be inferred.

Manuscript received February 2006; accepted June 2006. 